\title{
Modeling the Hysteresis Loop in Hard Magnetic Materials Using $\boldsymbol{T}(\boldsymbol{x})$ Model
}

\author{
M. Dośpiat*, M. Nabiatek, M. Szota, P. Pietrusiewicz, K. Gruszka, K. Beoch \\ Czestochowa University of Technology, Faculty of Materials Processing Technology and Applied Physics, Armii \\ Krajowej 19, 42-200 Czestochowa, Poland
}

\begin{abstract}
Possibility of decomposing the static hysteresis loop is demonstrated. The applied method is related with decomposition of experimentally obtained reversal magnetization curve on the reversible and irreversible components. Results are applicable in analysis of reversal magnetization processes in hard magnetic materials and for simulation of hysteresis loop using hyperbolic $T(x)$ model.
\end{abstract}

DOI: 10.12693/APhysPolA.126.170

PACS: 75.60.-d, 75.60.Jk, 75.50.Vv, 75.60.Ej

\section{Introduction}

In the available literature the $T(x)$ model is widely used to describe the hysteresis of soft magnetic materials [1-3]. There is an acute lack of scientific papers, in which are demonstrated possibilities of application of hyperbolic $T(x)$ model for description of hysteresis in hard magnetic materials.

Takacs and Mészáros in [2] have described a method for the decomposition of the hysteresis loop of soft magnetic materials, depending on content of the components, with different magnetic hardness (steels characterized by different values of saturation magnetization and coercivity). Each component has been described by single distribution function. In this approach the influence of physical processes on the reversal magnetization mechanism haven't been distinguished. Analysis of the influence of these processes has been carried out by Varga et al. [3]. In his work the magnetization has been separated into the components of magnetization processes associated with the rotation of the magnetization vector and the movement of domain walls.

In this paper authors are presenting decomposition of hysteresis loop of hard magnetic materials into the magnetization components, due to their type (reversible: rotation of magnetization vector motion of unpinned domain walls, bowing of strongly pinned domain walls, as well as irreversible: nucleation and growth of domains with reversed magnetization and pinning of domain walls).

\section{2. $T(x)$ model}

The shape of major hysteresis loop was described using $T(x)$ hyperbolic model, taking into account contribution of the reversible and irreversible processes, by the following mathematical equations:

*corresponding author; e-mail: mdospial@wp.pl

$$
\begin{aligned}
& m_{+n}=\sum_{k=1}^{n}\left(A_{k} \cdot f_{+k}+b_{n}\right), \\
& m_{-n}=\sum_{k=1}^{n}\left(A_{k} \cdot f_{-k}-b_{n}\right), \\
& f_{+k}=\tanh \left[a_{k} \cdot\left(h-a_{0 k}\right)\right], \\
& f_{-k}=\tanh \left[a_{k} \cdot\left(h+a_{0 k}\right)\right], \\
& b_{n}=\frac{1}{2} \sum_{k=1}^{n} A_{k}\left(f_{-k}-f_{+k}\right) \text { for } h=h_{m},
\end{aligned}
$$

where $m_{+n}$ and $m_{-n}$ are the normalized ascending and descending magnetization functions, respectively, $h$ is the field excitation, $a_{0 k}$ is the coercivity and $A_{k}$ is the amplitude of the $k^{t h}$ magnetization component, $a_{k}$ is the sheering factor and $b_{n}$ is the integration constant, while $h_{m}$ represents the maximum field excitation. The index $k$ refers to the individual magnetization component (both reversible and irreversible) and $n$ is their total number [3].

\section{Experiment methodology}

Studied sample was manufactured using melt-spinning method from $\mathrm{Sm}_{12.5} \mathrm{Co}_{66.5} \mathrm{Fe}_{8} \mathrm{Cu}_{13}$ alloy ingots. The obtained samples had a ribbon shape. Further, samples were annealed at $850{ }^{\circ} \mathrm{C}$ for $3 \mathrm{~h}$. Annealing process resulted in decomposition of meta-stable $\mathrm{SmCo}_{7}$ phase and formation of multiphase structure composed of $\mathrm{SmCo}_{5}$ and $\mathrm{Sm}_{2} \mathrm{Co}_{17}$ phases [4].

The static hysteresis loop and recoil curve, presented in this paper, were measured using LakeShore VSM with maximum external magnetic field of $2 \mathrm{~T}$. Sample used for magnetic measurements had a ribbon shape. The demagnetization field resulting from it's shape wasn't taken into account.

\section{Results and discussion}

Figure 1a presents hysteresis loop and recoil curve measured in demagnetization direction.

The recoil curve was used to decompose demagnetization curve into reversible and irreversible components 


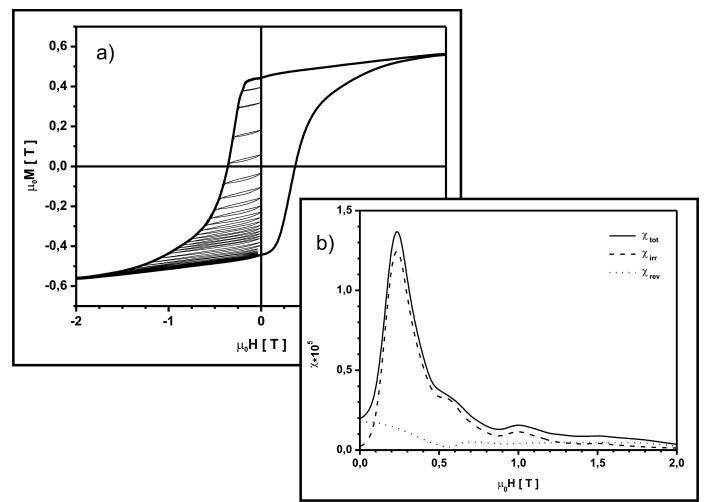

Fig. 1. Recoil curve measured along the demagnetization direction, and the determined on its basis b) the reversible, the irreversible and the total differential susceptibilities.

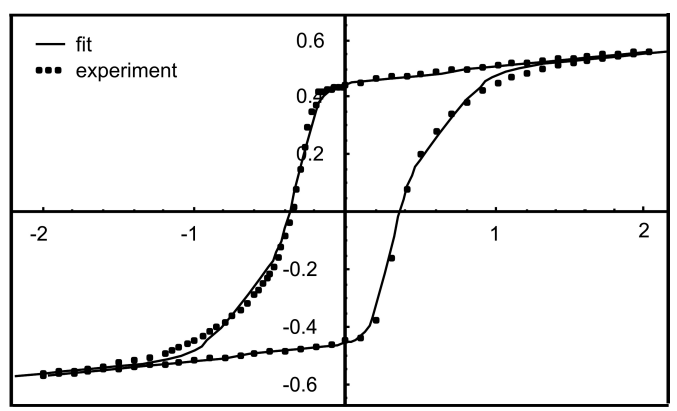

Fig. 2. Hysteresis loop obtained by application of $T(x)$ model, taking into account the presence of reversible and irreversible processes.

by the method described elsewhere [5]. In turn, these components were used to determine reversible and irreversible differential susceptibilities (Fig. 2b), and to describe the reversal magnetization mechanism occurring in the studied sample.

The reversal magnetization process in studied composite magnet, consisting of the $\mathrm{SmCo}_{5}$ and $\mathrm{Sm}_{2} \mathrm{Co}_{17}$ phases, is associated with pinning of domain walls on grain boundaries. On the irreversible differential susceptibility curve (Fig. $2 \mathrm{~b}$ - dash), three maxima for $0.232 \mathrm{~T}$, $0.558 \mathrm{~T}$ and $1.01 \mathrm{~T}$ were distinguished, which were attributed to three pining sites.

The reversible susceptibility curve had high initial value and decreased with applied field. Further, for external fields above $0.5 \mathrm{~T}$ a slight growth followed by slow fall with increasing external magnetic field was observed. The initial changes were attributed to the bowing of pinned domain walls, movements of unpinned domain walls in multi-domain grains and the rotations of magnetization vectors, while for stronger fields the reversible magnetization changes were dominated mainly by the rotation of magnetization vectors.

The analysis of susceptibility components helped to determine the two reversible and three irreversible processes that have a significant influence on the shape of the hysteresis loop $(n=5)$. For each process, the distribution functions were determined independently, using sech function and the $a_{0 k}$ (where $k=1-5$ ) parameters were determined. The obtained data were used as the initial parameters for simulation of hysteresis loop using $T(x)$ hyperbolic model. The simulated curve was compiled with data from the magnetic measurements and is shown in Fig. 2.

The area under three distributions, describing irreversible susceptibility changes and positions of their centers, were used to determine the influence of each pining site on coercivity $(0.37 \mathrm{~T})$ of sample, by the method described in [5]. The obtained results were as follows: $63 \%$ $(0.232 \mathrm{~T}) 29 \%(0.558 \mathrm{~T})$ and $8 \%(1.01 \mathrm{~T})$.

\section{Conclusions}

Recoil curve measured in demagnetization direction allow to determine reversal magnetization processes, shaping the hysteresis loop.

Data obtained from analysis of susceptibility curves for both types of processes allow to describe with good accuracy a hysteresis loop, using hyperbolic $T(x)$ model.

After decomposition of irreversible magnetization component, it is possible to evaluate influence of each pining site on coercivity of the sample.

\section{Acknowledgments}

This work was supported by the project of the Ministry of Science and Higher Education of Poland through Grant No. N N507 234940

\section{References}

[1] J. Takacs, Gy. Kovacs, L.K. Varga, J. Magn. Magn Mater. 320, e1016 (2008).

[2] J. Takacs, I. Meszaros, Physica B 403, 3137 (2008).

[3] L.K. Varga, Gy. Kovacs, J. Takacs, J. Magn. Magn. Mater. 320, L26 (2008).

[4] M.J. Dospial, M.G. Nabialek, M. Szota, T. Mydlarz, K. Oźga, S. Lesz, J. Alloys Compd. 536, S324 (2012).

[5] M. Dospial, D. Plusa, J. Magn. Magn. Mater. 330, 152 (2013).

[6] M. Dospial, M. Nabialek, M. Szota, D. Plusa, J. Alloys Compd. 509, S404 (2011). 